\title{
In Situ Characterization of Exposed E-Beam Resist Using Novel AFM Technique
}

\author{
H. Koop, ${ }^{*}$ M. Zech, * D. Schnurbusch, ${ }^{* *}$ M. Mueller, ${ }^{*}$ K. Karrai* and A. Holleitner** \\ * Attocube Systems AG, Koeniginstrasse 11a RGB, 80539 Munich, Germany \\ ** Walter Schottky Institute and Physics Department, TUM Garching, Germany
}

Pushing the size of e-beam lithography pattern to the nanometer range opens up new challenges for both lithography apparatus and their operators. Achieving highest resolutions requires a better understanding and control of the processes taking place in e-beam resists on the nanometer scale. New and reliable characterization techniques are necessary in order to develop next generation high resolution resists. For this purpose, we introduce a novel approach for the in-situ characterization of the impact of e-beam exposure on PMMA (poly-methyl methacrylate), using an atomic force microscope (AFM), fully integrated into an electron beam lithography (EBL) system. With ex-situ AFM data differing from in-situ measurements caused by the adsorption of water and subsequent swelling of the resist, we believe that only the in-situ approach delivers reliable data. We have implemented a tuning fork based AFM [1] into Raith's e_LiNE EBL system enabling simultaneous imaging of samples with both microscopes. All e-beam exposures are performed on a single sample without breaking vacuum. After finishing the exposure process, its impact on the PMMA is evaluated by scanning the topography of the exposed resist with AFM in non-contact mode.

In this paper we present the systematic investigation of two different resist systems, namely PMMA A6 with a thickness of 360nm and PMMA A2 with a thickness of 60nm, respectively. In both resists line-and-spaces pattern are exposed at different electron doses while keeping the acceleration voltage constant at $20 \mathrm{keV}$. Fig.1c) and 1d) show AFM phase and topography images of PMMA A2 as exposed to a $1 \mu \mathrm{m} / 2 \mu \mathrm{m}$ line-and-spaces pattern at a dose of $190 \mu \mathrm{C} / \mathrm{cm}^{2}$, indicating the shrinkage in resist thickness at exposed locations. In this specific example, exposed areas show a shrinkage of $0.46 \mathrm{~nm}$ compared to unexposed areas on the resist. The noise limit of the AFM apparatus in vertical direction can be estimated by subtracting two neighboring topographic line cuts yielding a value of $40 \mathrm{pm}$ as shown in Fig. 1e). With increasing exposure dose, the resist shrinkage increases linearly for both resist systems, whereby the shrinkage effect is more pronounced for thicker resists. This effect is displayed in Fig. 2a) for $360 \mathrm{~nm}$ thick PMMA A6 (black) and for 60nm thick PMMA A2 (grey). It is noteworthy that the resist shrinkage decreases upon reduction of the size of exposed pattern. This can be observed in Fig. 2b) which shows the resist shrinkage for line/space $=1 / 1$ pattern plotted as a function of the line width for PMMA A6 (black) and PMMA A2 (grey).

The presented in-situ characterization technique has crucial prediction capabilities due to the linear dependence of resist shrinkage on exposure dose. It allows the user to investigate the quality of exposure processes without developing any resist, allowing a step-wise proximity correction and reexposing underexposed spots. Compared to the traditional approach of imaging the post-processed resist in a scanning electron microscope (SEM) as shown in Fig. 1a), in-situ AFM delivers more physically valuable information, such as polymer grain size and distribution. The resulting line edge roughness of exposed pattern [2] can be investigated directly after exposure, excluding the impact of chemical processes. Therefore, the in-situ AFM has great potential to be used as a classification and verification tool for the development of new high resolution e-beam resists and for the exposure of ultra-small structures. 
References

[1] K. Karrai et al., US Patent 6006594.

[2] E. A. Dobisz et al., J. Vac. Sci. Technol. B 5 (1997) 2318.

a)

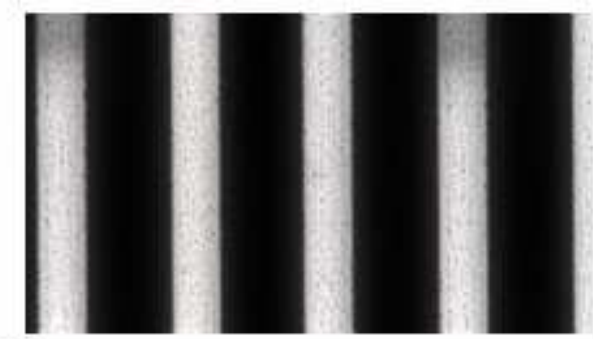

b)

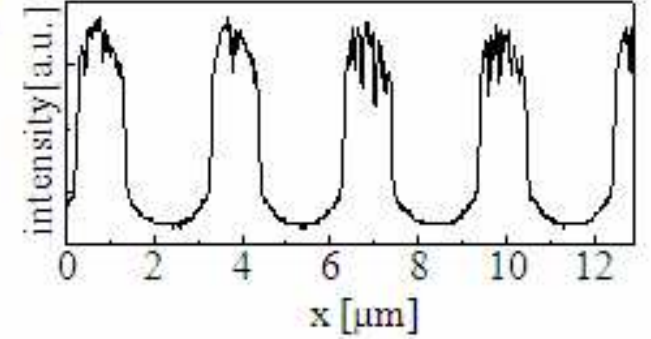

c)

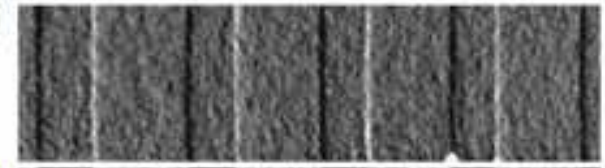

d)
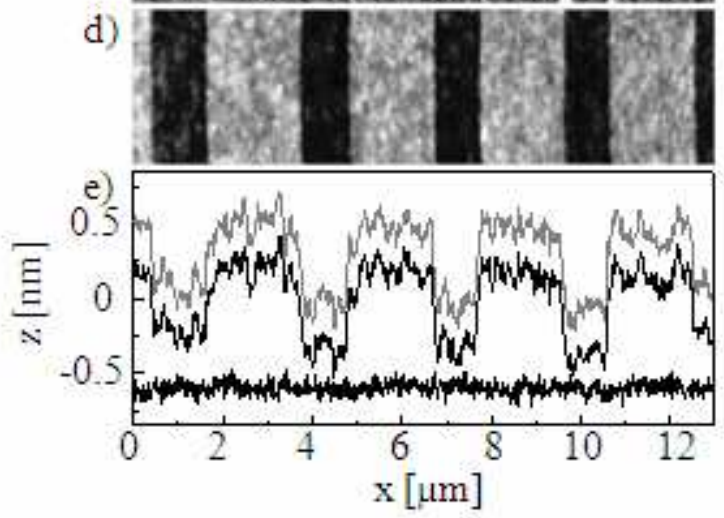

FIG. 1. a) Conventional SEM image of a resist after exposure and development. The contrast is given by the intensity of secondary electrons resulting from interactions of the electron beam with atoms at or near the surface of the sample. b) Intensity profile of a line cut taken at an arbitrary position within a). c), d) Phase and topography image of undeveloped resist measured in-situ by AFM. The topographic profiles of 2 neighboring lines cuts are shows in e), shifted vertically by 0.6 $\mathrm{nm}$ for better visualization. The bottom graph shows the subtraction of the (non-shifted) line cuts, demonstrating the performance of the AFM. The resulting vertical noise value is of order 40pm rms.
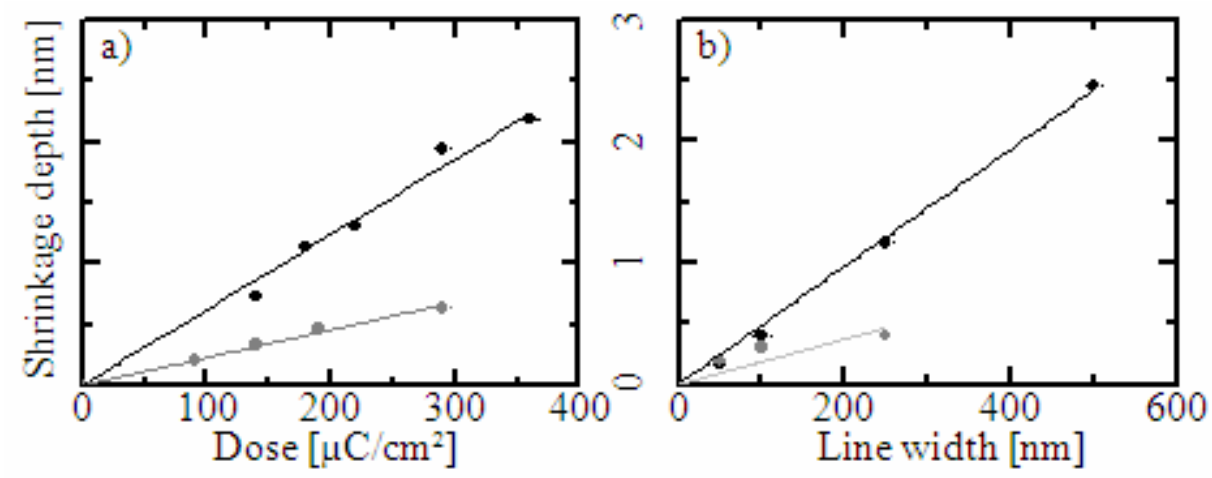

FIG. 2. Resist shrinkage after exposure plotted as function of exposure dose in a) and line width of line/space $=1 / 1$ pattern in b). Two different resists are investigated: PMMA A2 with a thickness of 60nm (grey) and PMMA A6 with thickness of 360nm (black). The linear fits serve as a guide for the eye. 